\title{
Current Trends and Issues Concerning the Competence of Mid-Career Community Health Nurses In Japan
}

\author{
Megumi Hattori' ${ }^{1}$ Estuko Yamada', Yoshika Suzaki ${ }^{2}$, Naoko Takayama ${ }^{3}$ \\ and Hiromi Ariyoshi ${ }^{1 *}$ \\ ${ }^{1}$ Saga University, Japan \\ ${ }^{2}$ The Japanese Red Cross Kyushu International College of Nursing, Japan \\ ${ }^{3}$ Yokkaichi Nursing and Medical University, Japan
}

Received: November 27, 2013; Accepted: December 10, 2013; Published: December 13, 2013

${ }^{*}$ Corresponding author: Hiromi Ariyoshi, Saga University, Japan, E-mail: ariyoshi@cc.saga-u.ac.jp

Key words: Community health nurses ability

The labor force participation rate of Japanese women by age group shows an M-shaped curve with the first descent beginning at age 25. While this trend is not seen in other countries such as the United States, Germany, or Sweden, the reasons for decline in Japan are resignation due to marriage or pregnancy, and devotion to the home and childcare. The Japanese government has set the numerical goal for raising the female employment rate (ages 25 through 44 ) at 73\%, the same rate as northern European countries. One of the key measures for this campaign focuses on "competency" and offers "support for rejoining the labor force", to include vocational training/research and cultivation of skills.

There are currently 55,262 public health nurses in Japan, 99\% of which are female. The child rearing generation is considered to be "mid-career public health nurses" with 6 to 20 years of work experience. A search of Japanese articles concerning community health nurses reveals a diverse interpretation of competency, and a survey conducted on community health nurses showed a difference in self-assessed competency verses superior-assessed competency. The results showed that those with higher selfratings received lower evaluation scores from their superiors. While exploring this interesting difference in competency evaluation would be a valid future research topic, our study focused on interviews with the superiors.

From our experience through this study, we learned that measuring or evaluating competency is a difficult task. If the community health nurse is unaware that they are "incapable", they are consequently obstructing their ability to improve. $60 \%$ of public health nurses are community health nurses who are regional government workers. Working in an environment where maternity and child care leave is easily accessible, it is necessary for community health nurses to continue working as specialists after their leave of absence, and to maintain a high level of competence throughout their career.

It is the desire of the authors of this study to assist in the creation of a work environment where child-rearing community health nurses can manifest their abilities. The Ministry of Health, Labor and Welfare (MHLW) describes the three types of competency as, basic ability, ability as a government worker, and ability as a specialist. We will begin by focusing on the three competencies listed by the MHLW and will continue to report on our results in the future. 